\title{
ON THE ORDER OF LINEAR HOMOGENEOUS GROUPS*
}

\author{
(FOURTH PAPER)
}

BY

\section{H. F. BLICHFELDT}

1. In the writer's theorems $\dagger$ on finite groups of linear homogeneous substitutions of determinant unity, a group of special nature called a self-conjugate (or invariant) subgroup $H$ plays an important rôle. There is a lack of completeness to these theorems due to the fact it has not been proved that $H$ is actually less than the transitive (irreducible) group $G$ in which, under certain conditions, it is contained; i. e., the groups $G$ and $H$ may be identical so far as the theorems are concerned. Account had to be taken of this fact in constructing the collineation-groups for the plane and space.t

The relation between $G$ and $H$ is as follows. Let the number of variables be $n$, and let $V$ be any substitution of $G$. The sum of the multipliers of $V$ (weight, characteristic) we shall indicate by $(V)$, which, therefore, represents the sum of $n$ roots of unity :

$$
(V)=\sum_{i=1}^{n} \alpha_{i} \beta_{i}
$$

Each of these roots we write as the product of one $\alpha_{i}$, whose index is prime to a given prime number $p$, and one $\beta_{i}$, whose index is a power of $p$. By $(V)_{p}$ we indicate the quantity obtained by replacing in $(V)$ every root $\beta_{i}$ by unity:

$$
(V)_{p}=\sum_{i=1}^{n} \alpha_{i}
$$

Then, under certain conditions, as mentioned above, there is in $G$ a self-conjugate subgroup $H$ whose substitutions $T$ have the property

from which, in particular,

$$
(V T)_{p} \equiv(V)_{p} \quad(\bmod p)
$$

$$
(T)_{p} \equiv n \quad(\bmod p)
$$

* Presented to the Society (San Francisco), September 24, 1910.

$\dagger$ The theorems chiefly concerned are as follows: Theorems 10 and 11 of the article On the Order of Linear Homogeneous Groups (Second Paper), these Transactions, vol. 5 (1904), pp. 315, 317 ; Theorem 14 of the article On the Order of Linear Homogeneous Groups (Supplement), ibid., vol. 7 (1906), p. 523. These two articles will be referred to by $L G I I$ and $L G S$ respectively. † Mathematische Annalen, vol. 60 (1905), p. 214, §16; vol. 63 (1907), p. 563, § 15 . 
2. The writer has recently been able to prove that $H$ (or more strictly, a modified form of it) is actually less than $G$ when this group is primitive, so that in all such cases $G$ cannot be a simple group. Furthermore, it follows that

$$
n \equiv 0 \quad(\bmod p) .
$$

Theorem 14 of $L G S$ may be modified to read :

TheOREM 14'. If for $n>1$ a primitive group $G$ in $n$ variables has an abelian subgroup $K$ of order $p^{a} \geqq p^{n}$, then $G$ will have an invariant subgroup $H$ which contains at least $p^{a-n+1}$ substitutions of $K$, but which does not contain $K$ entirely. If $T$ be any substitution of $H$, and $V$ any substitution of $G$ whose weight $(V)$ is zero, ${ }^{*}$ then

Moreover,

$$
(V T)_{p} \equiv 0 \quad(\bmod p)
$$

$$
(T)_{p} \equiv 0, \quad \text { and } \quad n \equiv 0 \quad(\bmod p) .
$$

Let the substitutions of $K$ be represented by $S_{0}^{y}=$ identity, $S_{1}, \ldots, S_{p^{a}-1}$.

In proving Theorem $14, L G S$, it was first proved that for at least $p^{a-n+1}$ substitutions of $K$, $\dagger$ say

$$
S_{0}, S_{1}, \cdots, S_{i}, \cdots,
$$

we have equations of the form

$$
\left(V S_{i}\right)-(V)+(1-\theta) X_{i}=0,
$$

$V$ being any substitution of $G, \theta$ a root of the equation

and $X_{i}$ a sum of the weights

$$
\theta^{p a}-1=0,
$$

$$
\left(j=0,1, \cdots, p^{a}-1\right),
$$

multiplied by integral functions of $\theta$, the numerical coefficients entering being integers or fractions whose denominators are prime to $p$.

Now let $V$ be any substitution of $G$ such that $(V)$ is zero. Then

$$
\left(V S_{i}\right)+(1-\theta) X_{i}=0
$$

for all substitutions $S_{i}$ of the series (1).

Consider all the weights (2).

* BuRnside has proved the following theorem: "In any irreducible group of linear substitutions of finite order, other than a cyclical group in a single variable, at least one of its characteristics is zero," Proceedings of the London Mathematical Society, ser. 2, vol. 1 (1903), p. 115.

$\dagger$ Attention is here called to an omission in LGS, page 524. First line below the matrix reads, "Now, to this matrix may be added $p^{a-m+1}$ rows $\cdots$ " This should read : "Now, to this matrix may be added $p^{a-m+1}-1$ rows $\cdots . "$ 
First, they may all vanish, whatever be the substitution $V$ chosen, so long as $(V)$ vanishes. By the arguments of $\S 7, L G I I$, all the substitutions of $K$ and all further substitutions $T$ of $G$ for which $(V T)$ vanishes, form a group $H$, self-conjugate in $G$. If $H$ had any weight $(W)$ which vanished, then every $(W T)$ would vanish, and therefore every $(T)$. But this is impossible, since $\left(S_{0}\right)=n$. Accordingly, every weight of $H$ is non-vanishing, and therefore $H$ is intransitive, by Burnside's Theoren. It follows by Theorem $8, L G I I$, and by Burnside's Theorem that $H$ is composed of similarity-substitutions.

Second, the weights (2) do not all vanish. By (3), some of them are divisible by $1-\theta$, the quotient being expressible as a linear function of a finite number of roots of unity, no numerical coefficient entering having a denominator which is divisible by $p$. Assume that all the weights (2) are divisible by $(1-\theta)^{k}$, whenever $V$ represents a substitution of $G$ whose weight is divisible by $(1-\theta)^{k}, k$ ranging through the values $0,1,2, \ldots, m$; but that the weights $(2)$ are not all divisible by $(1-\theta)^{m+1}$ whenever $(V)$ is divisible by $(1-\theta)^{m+1}$. In general, we should not expect $m$ to be greater than zero. Now, all the substitutions $T$ of $G$ for which

whenever

$$
(V T)=(1-\theta)^{m+1} X,
$$

$$
(V)=(1-\theta)^{m+1} Y,
$$

form an invariant subgroup $H$. To $H$ belongs the series (1), but not the entire group $K$.

Since $G$ is assumed to be primitive, $H$, if not composed of similarity-substitutions, must contain a substitution $W$ whose weight vanishes (Theorem 8, $L G I I$, and Burnside's Theorem). Then

$$
(W T)=(1-\theta)^{m+1} Z
$$

for every substitution $T$ of $H$; i. e., every

and therefore every

$$
(W T)_{p} \equiv 0 \quad(\bmod p),
$$

$$
(T)_{p} \equiv 0 \quad(\bmod p) .
$$

The Theorems 10 and 11, LGII, may be modified in like manner to read that the self-conjugate subgroup $H$ is less than $G$, the latter being primitive. In addition,

$$
(T)_{p} \equiv 0 \quad(\bmod p)
$$

for every substitution of $H$.

3. One of the most important problems in the theory of linear homogeneous groups is the determination of the maximum order; $i$. e., the fixing of a superior limit to $\lambda$ in Jordan's Theorem, the number of variables being given. The 
limit known * can now be greatly reduced in special cases. Let $G$ be a primitive group, $n$ the number of variables, and $p$ a prime. Then, using Theorem $14^{\prime}$ in conjunction with Theorem $9, L G I I$, we can prove the

CoRollary. If $p$ and $n$ are prime to each other, the highest power of $p$ which divides the order of $G$ must divide $n ! p^{n-1}$.

If $n=p$, an invariant subgroup $H$ (assumed to contain and to be greater than the group of similarity-substitutions of order $p$ ) must be of order $p^{k}$ and cannot be abelian, $G$ being assumed primitive. Writing $H$ in monomial form we readily find that, if $k>3$, it possesses one, and only one, invariant of degree $p$ which can be factored into $p$ linear factors. In such a case $G$ cannot be primitive. Hence, $H$ is of order $p^{3}$ (or $p^{2}$ when considered as a collineation-group), being generated by the substitutions

$$
\begin{aligned}
& A: x_{1}^{\prime}=x_{1}, x_{2}^{\prime}=\theta x_{2}, \cdots, x_{p}^{\prime}=\theta^{p-1} a_{p} ; \frac{\theta^{p}-1}{\theta-1}=0 ; \\
& B: x_{1}^{\prime}=x_{2}, x_{2}^{\prime}=x_{3}, \cdots, x_{p}^{\prime}=x_{1} .
\end{aligned}
$$

The order of $G$ is a factor of $\left(p^{2}-1\right) p \cdot p^{2}$ when considered as a collineationgroup. The corollary above is therefore true also when $n=p>2$.

Theonem 17. Let $G$ be a primitive collineation-group in $n$ variables, $n$ being a prime $>1$. Then the order of $G$ is a factor of

$$
n !(2 \cdot 3 \cdots \cdots \cdot p \cdot \cdots)^{n-1},
$$

$2,3, \cdots, p, \cdots$ being the different primes not greater than the greater of the numbers $4 n-3,(n-2)(2 n+1)+\dagger$ The only exception is the octahedral group of order 24 in two variables.

It will be noticed that a transitive group in a prime number of variables $n$ is either primitive or of monomial type. In either case, the corresponding Jordan factor $\lambda$ must divide the number (4) if $n>2$.

* See LGII, pp. 310, 320-321, and On Imprimitive Linear Homogeneous Groups, these Transactions, vol. 6 (1905), p. 232, for an upper limit for all transitive groups. ScHuR has given an upper limit when the weights belong to a given field: Sitzungsberichte der Kgl. Preussischen Akademie der Wissenschaften, 1905, p. $77 \mathrm{ff}$.

†LGS, p. 528. 